\title{
Ligament Formation Induced by Cavitation in a Cylindrical Nozzle"
}

\author{
Akira SOU**, Muhammad Ilham MAULANA ${ }^{* *}$, Shigeo HOSOKAWA ${ }^{* *}$ \\ and Akio TOMIYAMA** \\ ${ }^{* *}$ Graduate School of Engineering, Kobe University, \\ 1-1, Rokkodai, Nada, Kobe, 657-8501, Japan \\ E-mail: sou@mech.kobe-u.ac.jp
}

\begin{abstract}
Cavitation in a cylindrical nozzle and liquid jets discharged from the nozzle are simultaneously visualized using a high-speed camera to investigate the mechanism of liquid jet atomization induced by the fully-developed cavitation. Three mirrors are used to capture the front view of liquid jet interfaces and the side view of cavitation clouds within a frame of the camera. The high-speed visualization confirms that the collapse of a cavitation cloud near the nozzle exit induces a ligament formation not only for 2D nozzles but also for cylindrical nozzles. The visualization also finds that the collapse of a large cavitation cloud tends to cause the formation of a large ligament, while that of a small cloud results in a small ligament.
\end{abstract}

Key words: Cavitation, Cylindrical Nozzle, Injector, Atomization, Ligament

\section{Introduction}

In various industrial fields efforts have been made to develop atomizers, which can produce fine droplets. In 1959, Bergwerk ${ }^{(1)}$ pointed out that cavitation occurs in a nozzle of pressure atomizers and it strongly affects liquid jet behavior. Then, Hiroyasu et al. (2) showed that atomization of a liquid jet is promoted when cavitation is fully-developed in a nozzle (supercavitation ${ }^{(3)}$ ). Thus, a number of studies have been carried out to observe cavitation in nozzles of various pressure atomizers ${ }^{(3)-(5)}$. Attempts have been also made to control and utilize cavitation to produce fine droplets ${ }^{(6)}$.

Once we clarify the mechanism of atomization induced by the fully-developed cavitation, we will be able to develop new atomizers in which the mechanism is utilized more efficiently. Hence, a number of studies have been conducted to investigate the mechanism. Wu et al. ${ }^{(7)}$ reported that vorticity in the boundary layer in a nozzle plays an important role in the ligament formation at the liquid jet interface. He and Ruiz ${ }^{(8)}$ measured liquid velocity inside a two-dimensional (2D) nozzle using a laser Doppler velocimetry (LDV). Oda and Yasuda ${ }^{(9)}$ applied a particle tracking velocimetry (PTV) to a cavitation flow in a 2D nozzle. Both experiments showed that a strong turbulence is produced near the cavitation zone, which might contribute to atomization. These experiments were, however, not conducted in the supercavitation regime.

Recently the authors ${ }^{(10)}$ have measured liquid velocity in a $2 \mathrm{D}$ nozzle in supercavitation using LDV, and found that a strong turbulence is produced near the exit of the nozzle. We also carried out simultaneous visualization of supercavitation and liquid jets using a high-speed camera ${ }^{(11)}$. The LDV measurement and the high-speed visualization for the $2 \mathrm{D}$ nozzle confirmed that in the supercavitation regime cavitation bubble clouds are shed and collapse near the exit and a strong turbulence is induced at the trace of the cavitation cloud. 
When the strong turbulence comes out of the nozzle, a ligament which initiates atomization is formed. The ligament formation induced by the cavitation cloud will be briefly introduced in the next section. The mechanism of atomization by cavitation in the case of cylindrical nozzles, however, remains unknown due to the difficulty in the visualizations of cavitation in a cylindrical nozzle and a liquid jet with a complex three-dimensional configuration.

In this study cavitation in a cylindrical nozzle and a liquid jet discharged from the nozzle are simultaneously visualized using a high-speed camera in order to investigate cavitation in a cylindrical nozzle and its effects on atomization.

\section{Ligament formation induced by cavitation in two-dimensional nozzle}

Let us briefly explain the mechanism of atomization induced by the supercavitation in a $2 \mathrm{D}$ nozzle ${ }^{(11)}$. Figure 1 shows the high-speed images of cavitation in the 2D nozzle and liquid jet interfaces. The image of the liquid jet is slightly shifted in the lateral direction by an optical setup. The frame rate is $20000 \mathrm{fps}$. Cavitation clouds are shed and collapse near the nozzle exit. When the trace of the cavitation cloud comes out of the nozzle, a ligament is formed. Figure 2 illustrates the ligament formation induced by the cavitation cloud in a $2 \mathrm{D}$ nozzle.

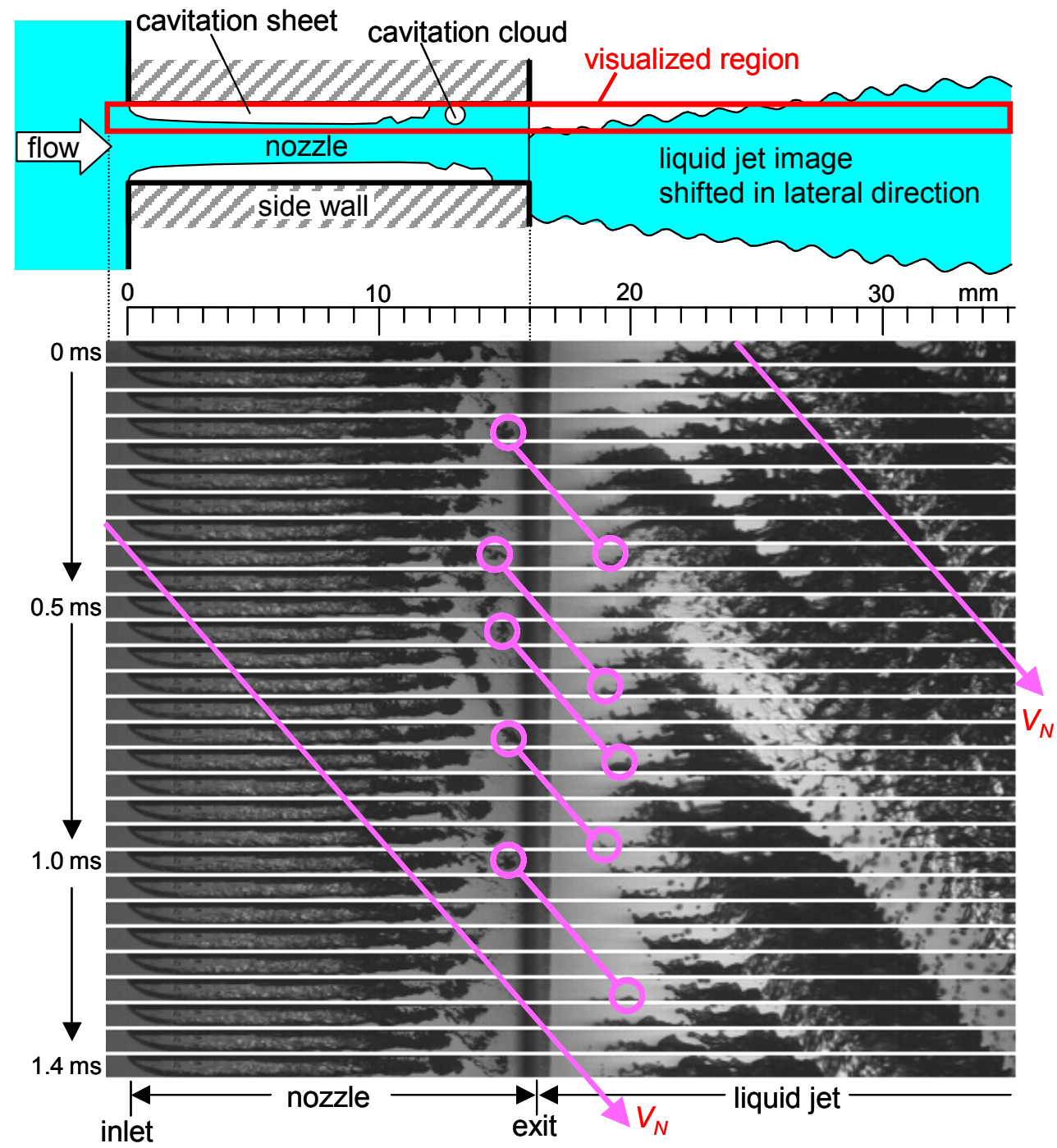

Fig. 1 Cavitation clouds in the 2D nozzle and the ligament formation (20000 fps) ${ }^{(11)}$ 


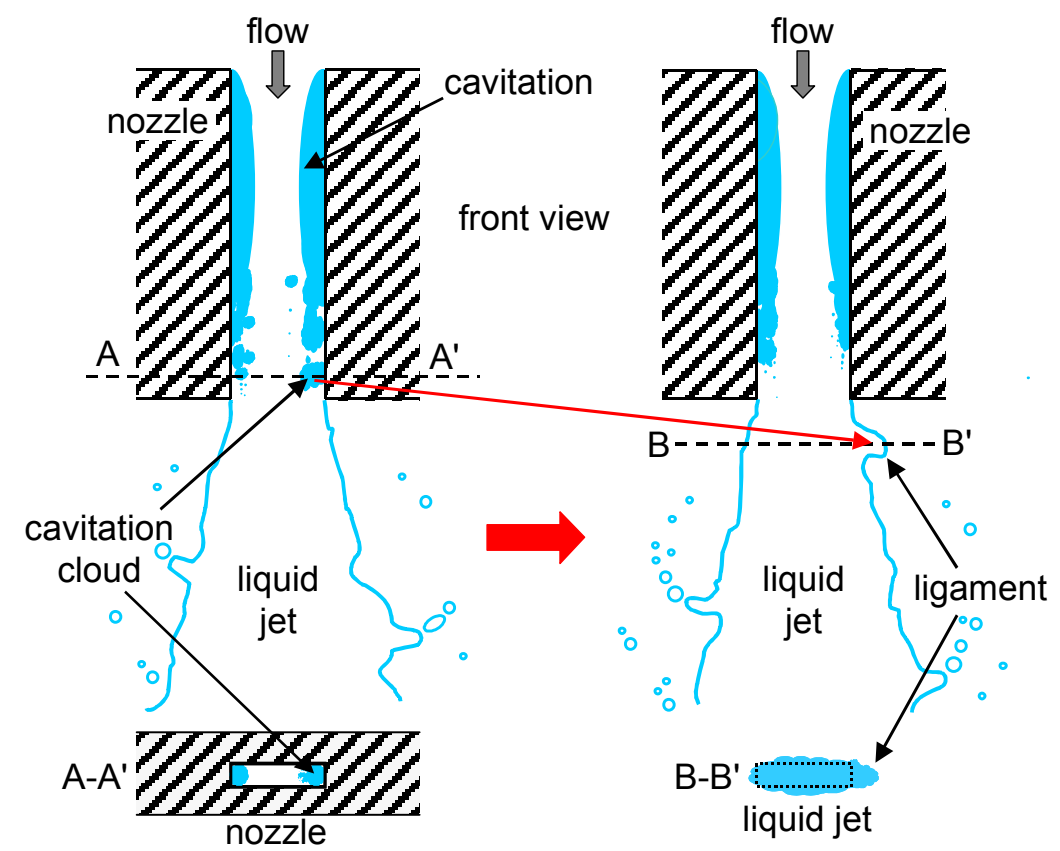

(a) before the collapse of a cavitation cloud

(b) after the collapse of the cavitation cloud

Fig. 2 Ligament formation induced by cavitation in a two-dimensional nozzle

\section{Hypothesis: ligament formation by cavitation in cylindrical nozzle}

Figure 3 (a) shows the image of supercavitation in the 2D nozzle and a liquid jet. Neither cavitation in the 2D nozzle nor the liquid jet is a bilateral symmetry. Note that there are two cavitation sheets in the upper half of the nozzle and two side walls in the case of 2D nozzles. On the other hand, there are only one circular side wall and one cavitation sheet developed along the wall in the case of cylindrical nozzles. Hence, whether or not the same process, i.e., the shedding and collapse of cavitation clouds and the ligament formation, takes place in cylindrical nozzles remains unsolved. Figure 3 (b) shows the image of supercavitation in a cylindrical nozzle of $4 \mathrm{~mm}$ in inner diameter and a liquid jet. The liquid jet is not axisymmetric.

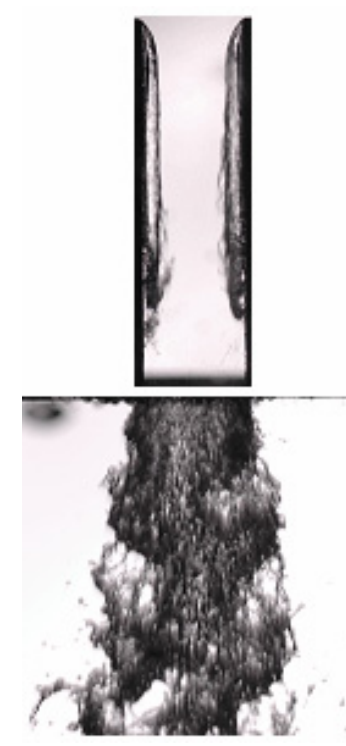

(a) 2D nozzle
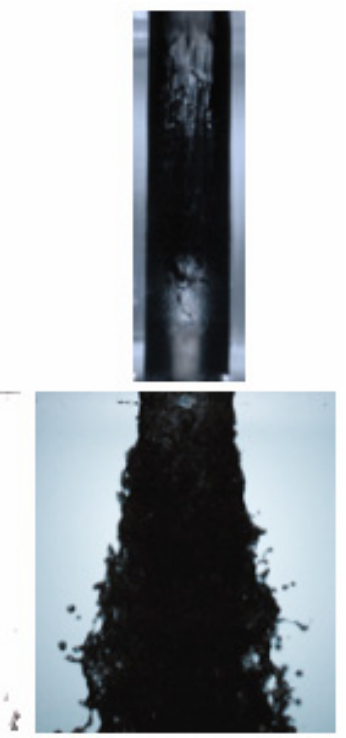

(b) cylindrical nozzle

Fig. 3 Asymmetric behaviors of cavitation in a nozzle and a liquid jet 
Based on the fact that cavitation clouds in the 2D nozzle induce ligament formation, we propose the following hypothesis for cylindrical nozzles. As shown in Fig. 4 (a), we assume that clouds of cavitation bubbles are formed at the skirt of the annular cavitation sheet, and a ligament is ejected at the trace of the cavitation cloud as illustrated in Fig. 4 (b).

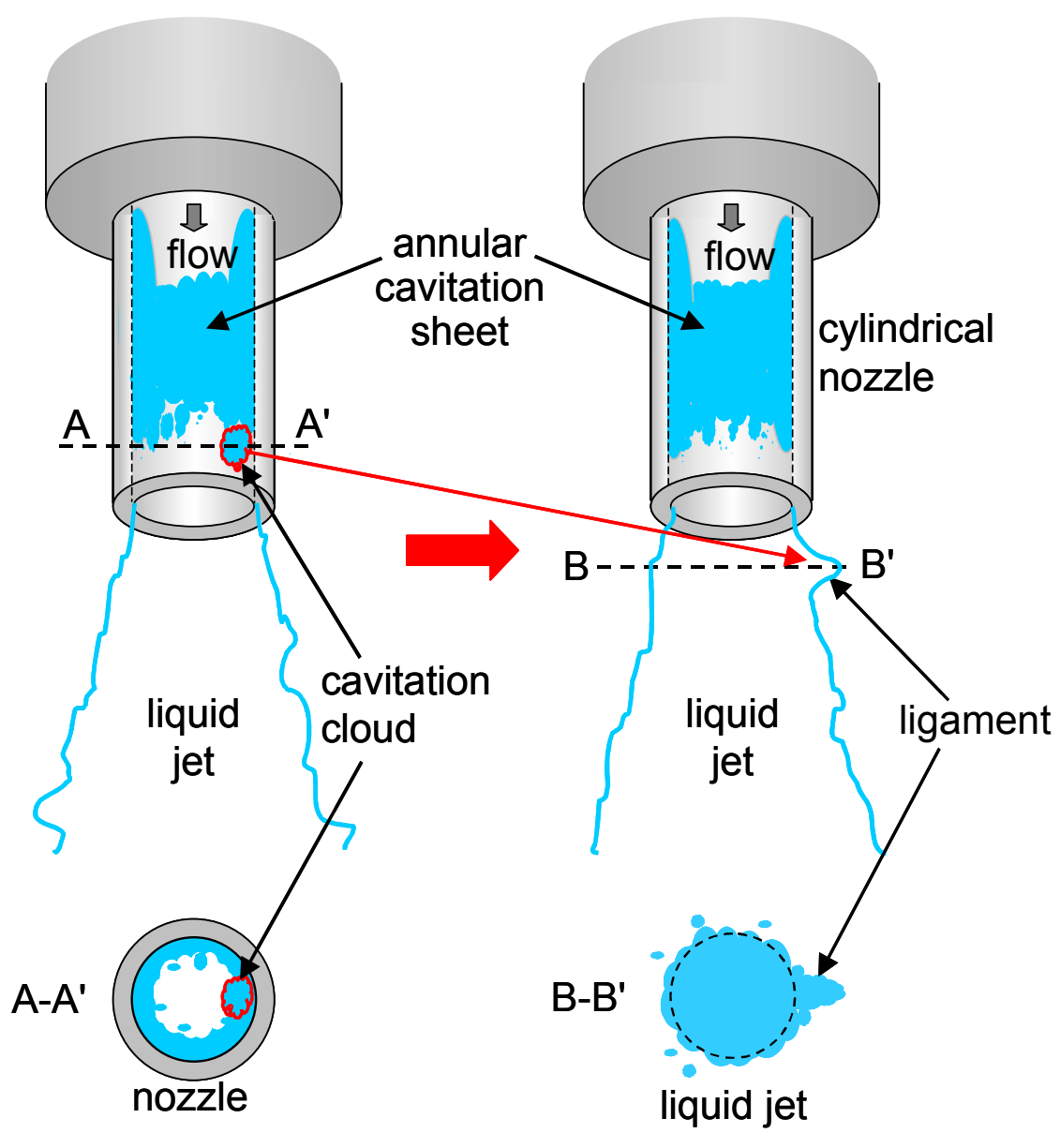

(a) before the collapse of a cavitation cloud

(b) after the collapse of the cavitation cloud

Fig. 4 A hypothesis: ligament formation induced by cavitation clouds in a cylindrical nozzle

\section{Experimental setup}

To examine whether the hypothesis is true or not, cavitation in a cylindrical nozzle and liquid jets were observed using a high-speed camera. Figure 5 shows the schematic of the experimental setup. A filtered tap water at room temperature was injected from the nozzle into ambient air. As shown in Fig. 6, the nozzle diameter $D$, the nozzle length $L$ and the sac diameter were 4, 16 and $32 \mathrm{~mm}$, respectively. The nozzle was made of transparent acrylic resin. The front and back sides of the outer surface of the nozzle were flattened for visualizations. First, the images of cavitation in the nozzle and liquid jets were taken using a digital camera (Nikon, D70, 3008 x 2000 pixels) and a flash lamp (Nissin Electronic, MS-1000 \& LH-15M, duration $=4 \mu \mathrm{s}$ ).

Figure 7 shows the experimental setup for the high-speed simultaneous visualization of cavitation and ligament formation. A high-speed camera whose frame rate was 5000 or 10000 fps was used (Redlake, MotionPro, HS-1, 64 x 1280 pixels for 10000 fps). As shown in Fig. 7 (a), a possible cavitation cloud which is formed near the right end of the inner surface cannot be observed from the front due to optical refraction at the wall surface. 
Hence, the side view of the nozzle was taken using the mirror A to visualize the cavitation cloud. The possible ligament formation induced by the cavitation cloud was observed from the front. To capture the cavitation clouds and the ligaments in a frame of $64 \mathrm{x} 1280$ pixels and to match the optical distance to the cavitation clouds and that to the ligaments, the front view of the ligaments was obtained using the mirrors $\mathrm{B}$ and $\mathrm{C}$ as shown in Fig. 7 (b).

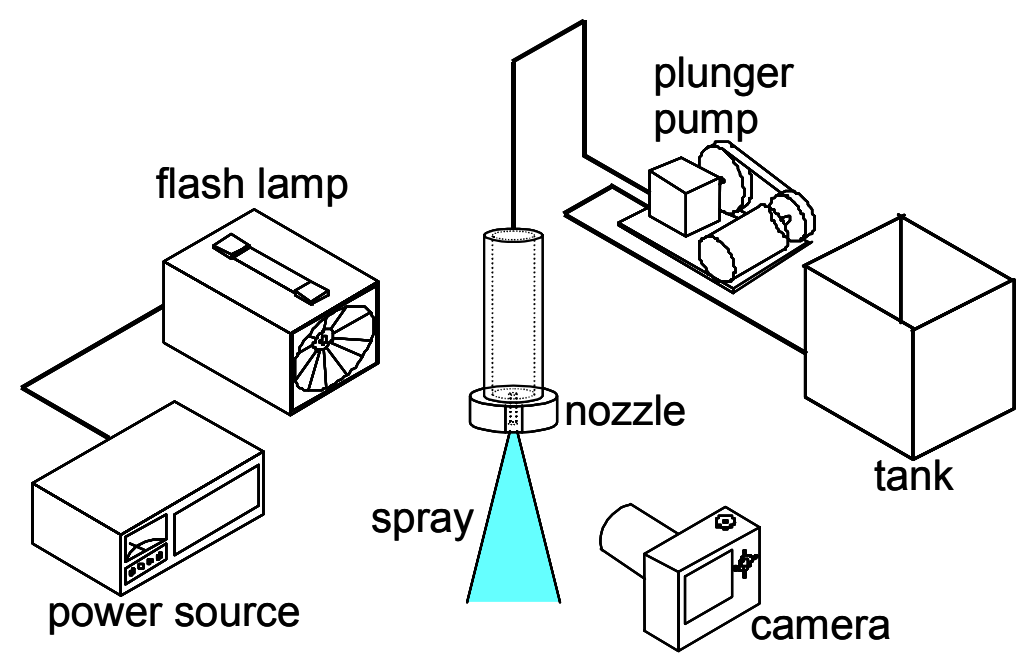

Fig. 5 Schematic of experimental setup

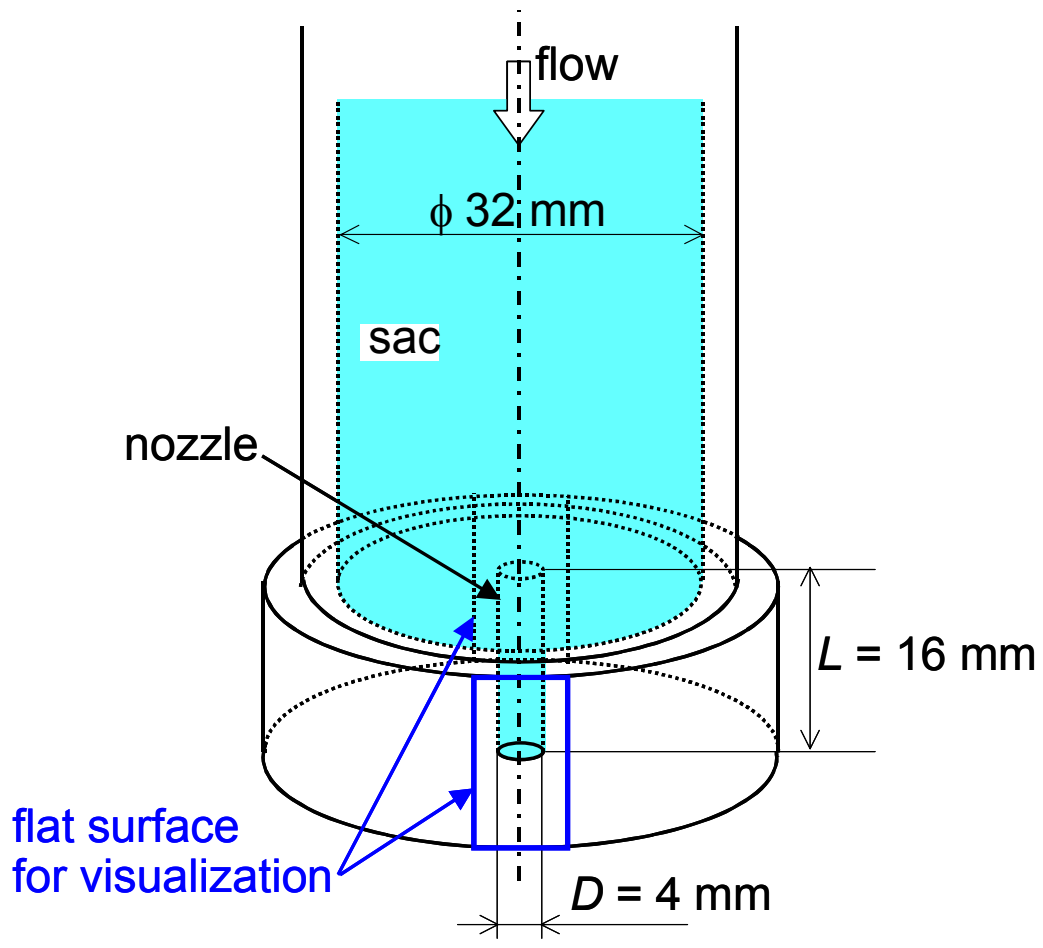

Fig. 6 Schematic of the cylindrical nozzle 


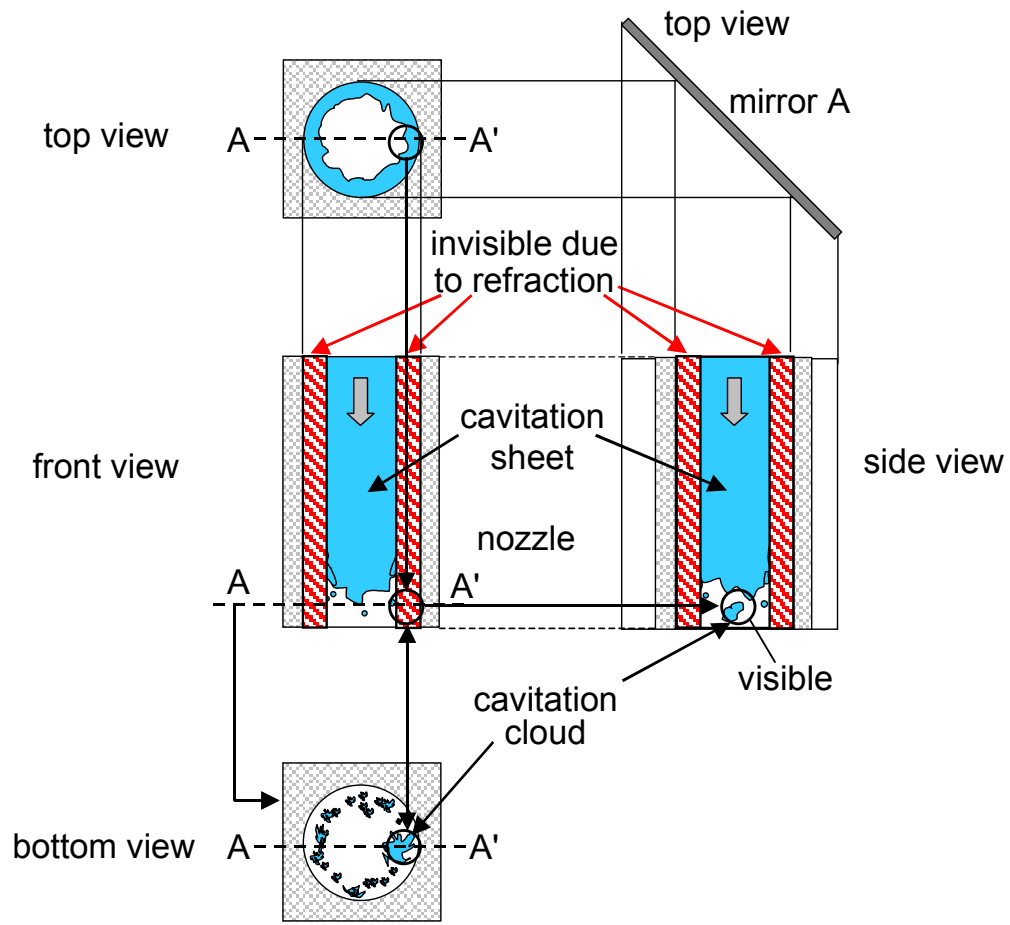

(a) visualization of cavitation cloud in a nozzle from right side

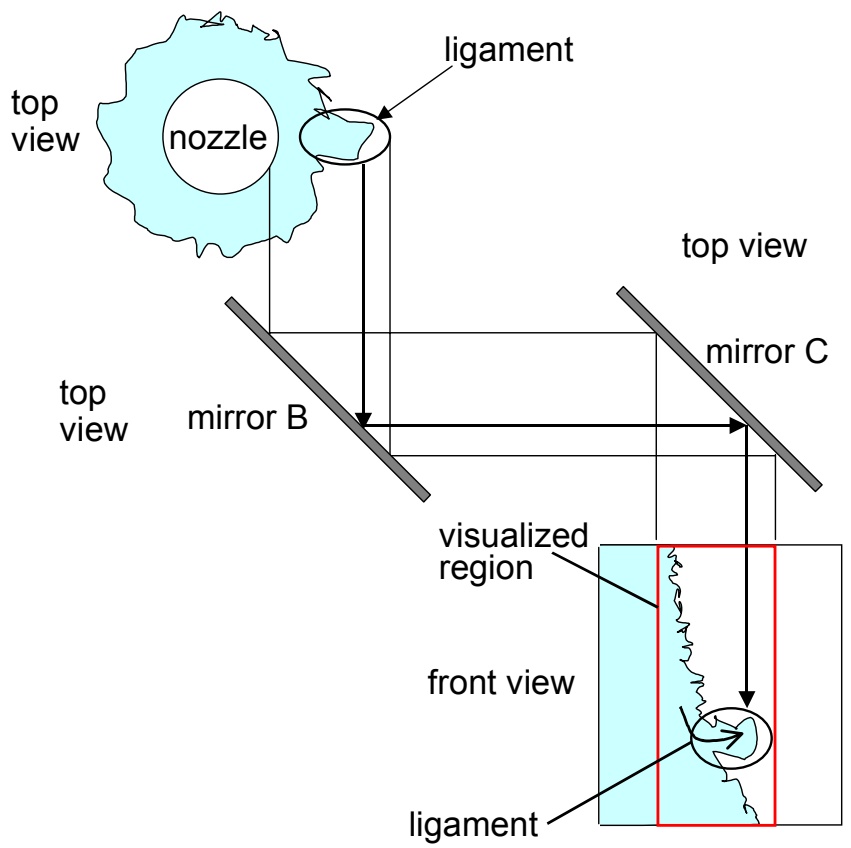

(b) visualization of ligament formation from the front

Fig. 7 Experimental setup for a simultaneous visualization of cavitation and ligament formation

\section{Results and discussion}

Figure 8 shows the images of cavitation in the cylindrical nozzle and liquid jets in no cavitation, developing cavitation, supercavitation and hydraulic flip. In developing cavitation, bubbles appear in the upstream half of the nozzle and the liquid jet is wavy. We call the flow "supercavitation" when a sheet cavitation covering most part of the nozzle wall is formed. In this case the liquid jet is atomized (spray). In hydraulic flip, the liquid flow separated at the inlet does not reattach to the nozzle wall, and the jet is smooth. These flow patterns shown in Fig. 8 are consistent with those in previous observations ${ }^{(2)-(6),(11)}$. 


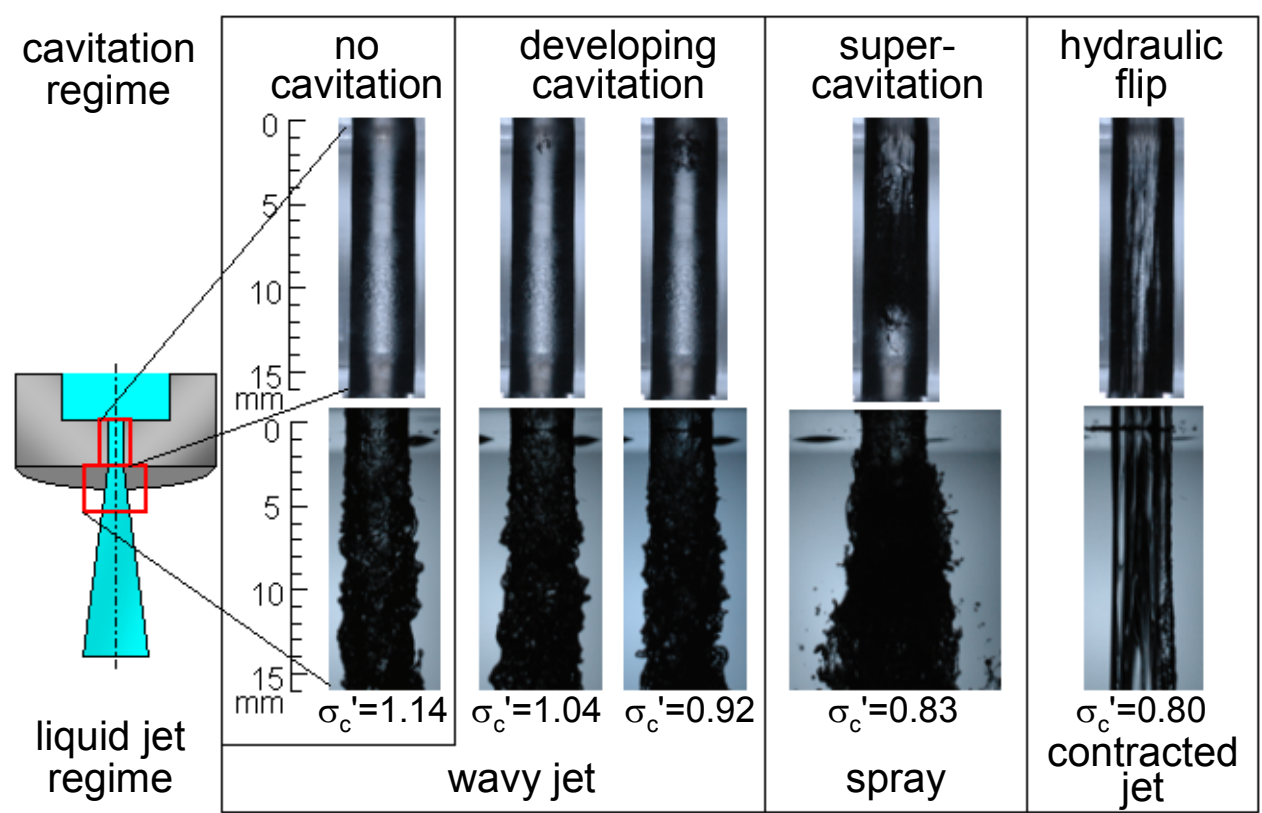

Fig. 8 Cavitation and liquid jet flow patterns

In Fig. 8 the cavitation number $\sigma_{\mathrm{c}}{ }^{\prime}$ is used as an indicator, which is defined by ${ }^{(12)}$

$$
\sigma_{c}{ }^{\prime}=\frac{\frac{1}{2} \rho V_{c}^{2}+P_{c}-P_{v}}{\frac{1}{2} \rho V_{c}^{2}}
$$

where $\rho$ is the liquid density, $V_{c}$ the mean velocity at the vena contracta in the nozzle, $P_{c}$ the pressure at the vena contracta, and $P_{v}$ the vapor saturation pressure. By evaluating $P_{c}$ using the Bernoulli's equation and introducing the contraction coefficient $C_{c}, \sigma_{\mathrm{c}}{ }^{\prime}$ is given by

$$
\sigma_{c}{ }^{\prime}=C_{c}^{2}\left[\frac{P_{b}-P_{v}}{\frac{1}{2} \rho V_{N}^{2}}+\frac{\lambda L}{D_{H}}+1\right]
$$

where $P_{b}$ is the back pressure (ambient pressure), $V_{N}$ the mean velocity in the nozzle, $\lambda$ the friction factor, and $D_{H}$ the hydraulic diameter of the nozzle. The value of $C_{c}$ is 0.61 for the present nozzle ${ }^{(13)}$, since it has a sharp inlet edge and the sac diameter is much larger than the nozzle diameter $D\left(=D_{H}\right)$. The $\lambda$ is given using the Blasius equation.

Figure 9 shows the dimensionless cavitation length $L^{*}$, which is defined as the ratio of the mean streamwise length $L_{c a v}$ of the cavitation zone to the nozzle length $L$, against the cavitation number $\sigma_{\mathrm{c}}{ }^{\prime}$. The experimental data for cylindrical nozzles with different sizes and shapes ${ }^{(14)}$ are also plotted in the figure. The present nozzle shows the same characteristics as the nozzles of smaller diameters.

Figure 10 shows high-speed images of cavitating flows in the cylindrical nozzle at $\sigma_{c}{ }^{\prime}=$ 0.83 (supercavitation). The visualization confirms that cavitation clouds are shed and collapse near the exit. As shown in Fig. 11, collapsing cavitation clouds sometimes take horseshoe shapes. 


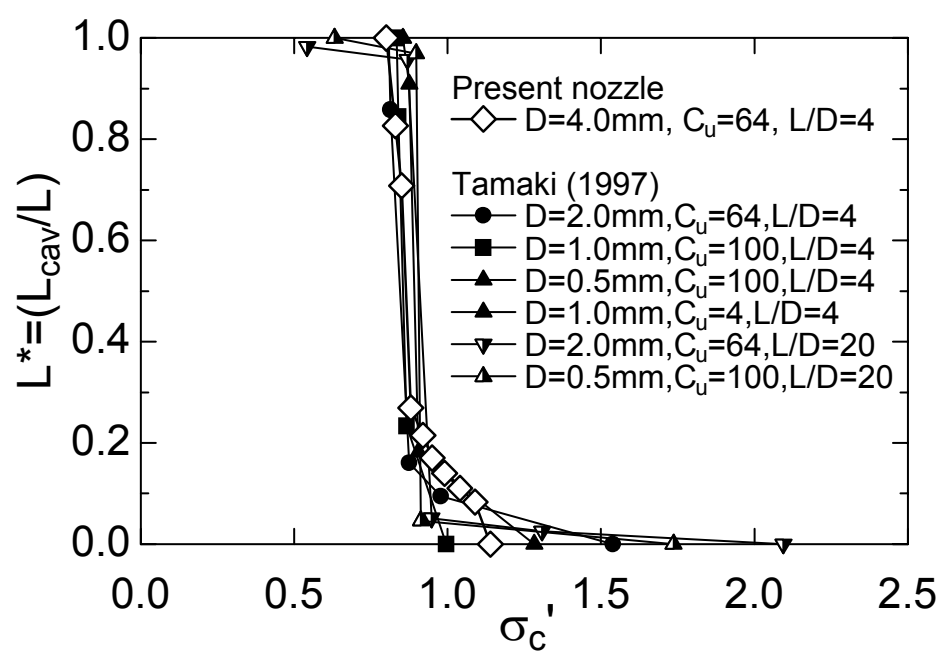

Fig. 9 The dimensionless cavitation length $L^{*}$ against the cavitation number $\sigma_{c}{ }^{\prime}$ ( $C_{u}$ is the ratio of the cross-sectional area of the sac to that of the nozzle)

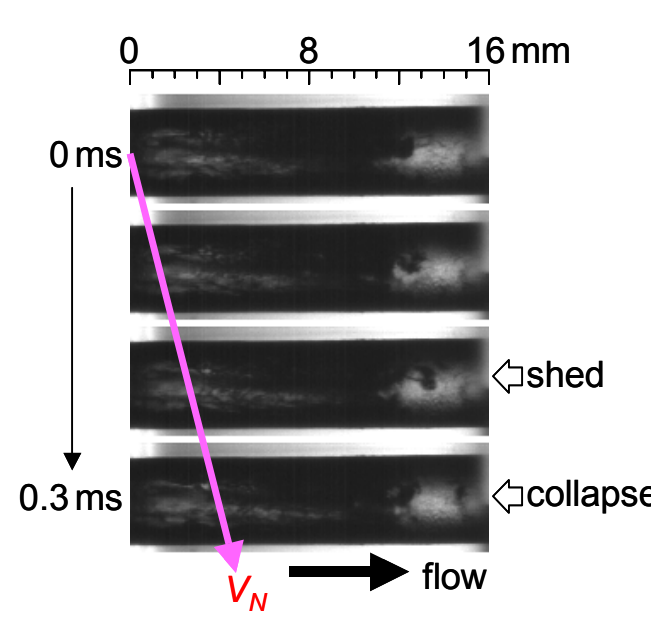

(a) shedding and collapse of a cavitation cloud

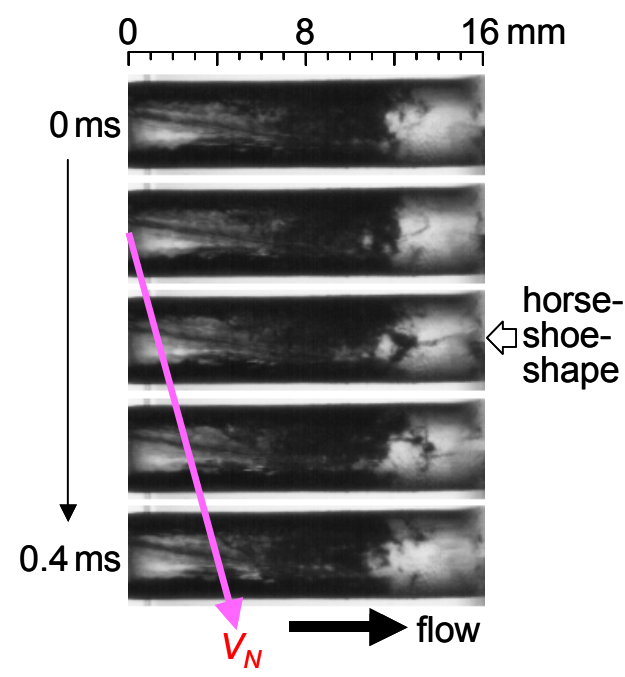

(b) a collapsing cavitation cloud

Fig. 10 Cavitation clouds in a cylindrical nozzle $\left(\sigma_{c}{ }^{\prime}=0.83,10000 \mathrm{fps}\right)$
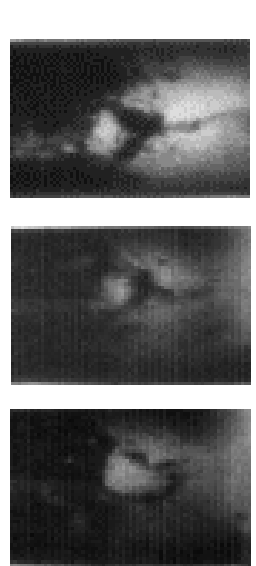

(a) five examples of collapsing cavitation clouds
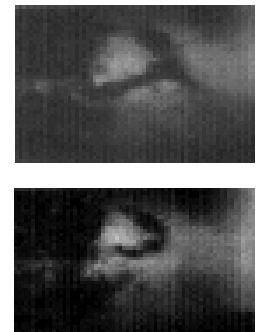

horseshoe-shaped cavitation cloud

Fig. 11 Horseshoe-shaped cavitation clouds

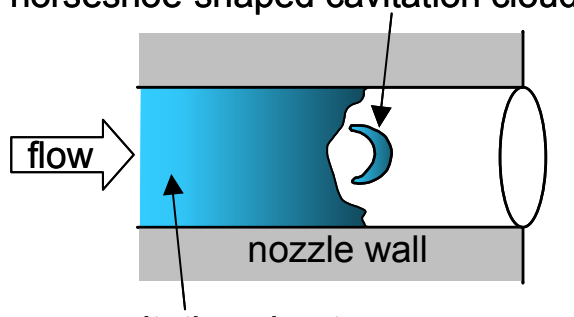

cavitation sheet

(b) a horseshoe-shaped cavitation cloud 
Figures 12 shows high-speed images taken from the front without using the mirrors. As shown in Fig. 12 (a), liquid jets discharged from the cylindrical nozzle are not axisymmetric, which indicates that ligaments are formed randomly in the azimuthal direction. The observation of three thousand sequential images captured from the front (e.g., Fig. 12 (b)) cannot make clear the causal relationship between the cavitation cloud and the ligament formation.

High-speed images of the side view of cavitation and the front view of liquid jet interfaces captured using the present experimental setup (Fig. 7$)$ are shown in Fig. $13\left(\sigma_{c}{ }^{\prime}=\right.$ $0.83,10000 \mathrm{fps})$. The visualization clearly indicates the causal relationship between the cavitation cloud and the ligament formation. We observed 1013 cavitation clouds in more than 6000 images, from which the ligament formation was observed 771 times. Hence, the probability of the ligament formation was $76 \%$. The strong relationship between the cavitation cloud and the ligament formation confirms the validity of the hypothesis, that is, the collapse of a cavitation cloud near the nozzle exit induces the ligament formation not only for 2D nozzles but also for cylindrical nozzles.

Note that the probability was higher than $94 \%$ in the case of the $2 \mathrm{D}$ nozzle, i.e., 498 collapses of cavitation clouds resulted in 471 formations of ligaments. The reason of the lower probability in the case of the cylindrical nozzle may be due to ligament ejections, which are invisible from the front as illustrated in Fig. 14. As shown in Fig. 15, large cavitation clouds tend to induce large ligaments, and small clouds induce small ligaments.

There might be an argument that the ligament formation is caused not by the collapse of cavitation clouds but by a possible swirling flow in a jet. However, we have already confirmed that the collapse of cavitation clouds causes the ligament formation for $2 \mathrm{D}$ nozzles in which no swirling flow occurs ${ }^{(11)}$. Hence, the swirling flow is not the cause of the ligament formation in the cylindrical nozzle.

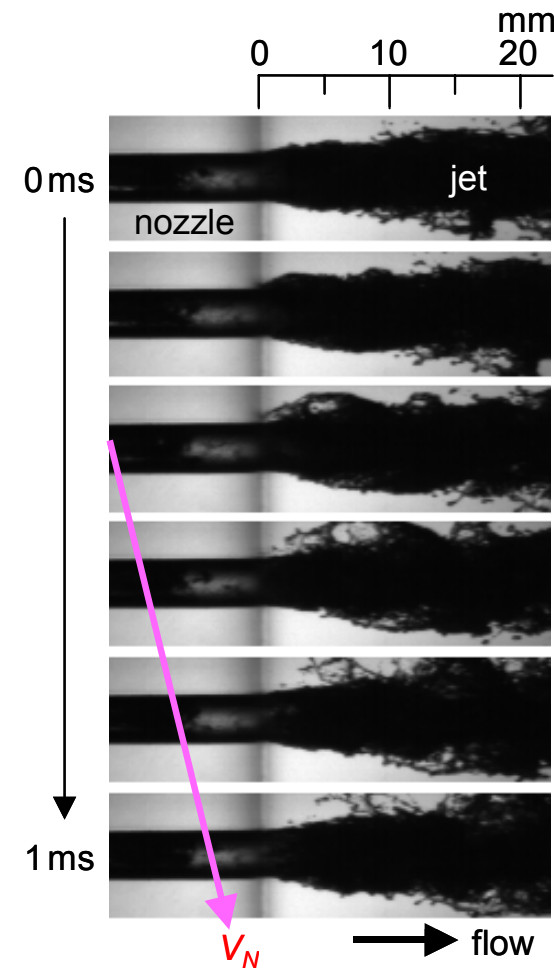

(a) asymmetric liquid jet

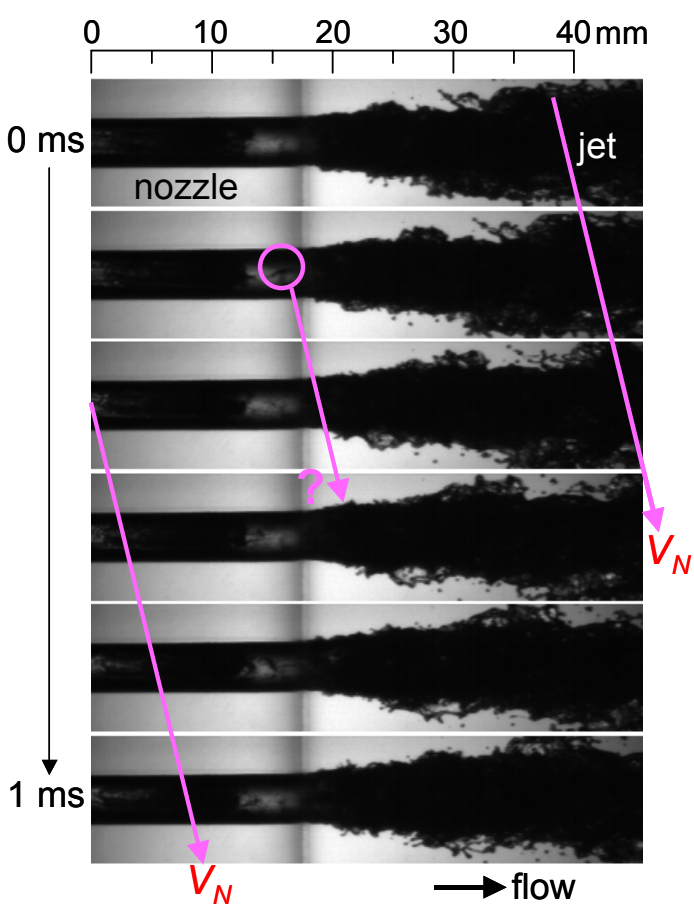

(b) no ligament in the trace of a cloud

Fig. 12 Front views of cavitation and liquid jets $\left(\sigma_{c}{ }^{\prime}=0.83,5000 \mathrm{fps}, 128 \times 1280\right.$ pixels $)$ 

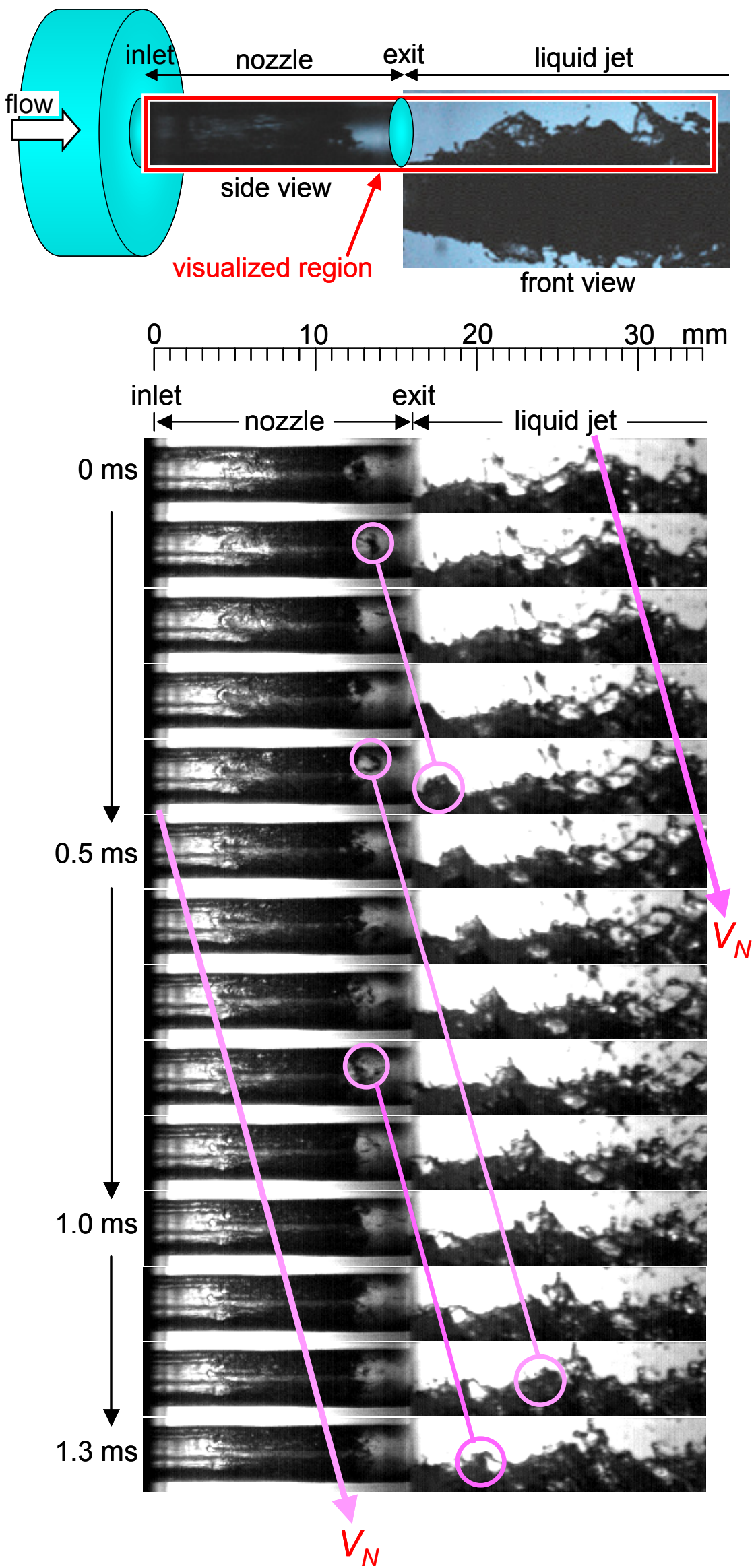

Fig. 13 Cavitation clouds in a cylindrical nozzle and liquid jet deformation $\left(\sigma_{c}{ }^{\prime}=0.83,10000 \mathrm{fps}\right)$ 

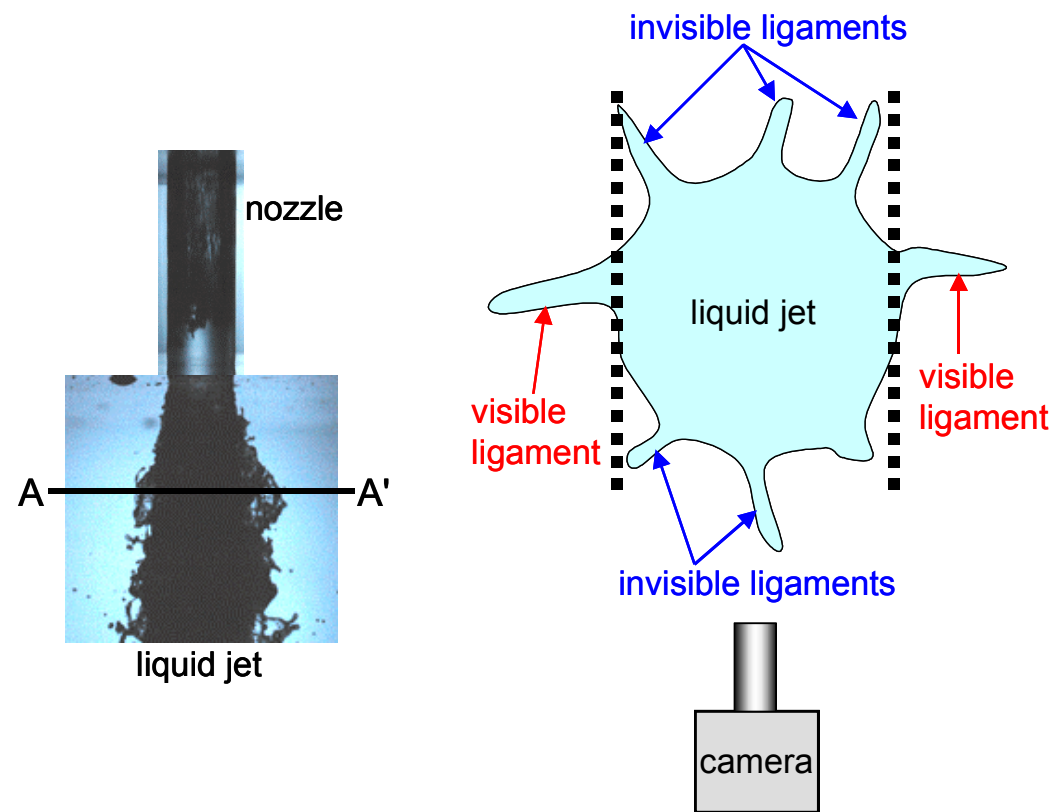

Fig. 14 Visible and invisible ligaments (cross section A-A')

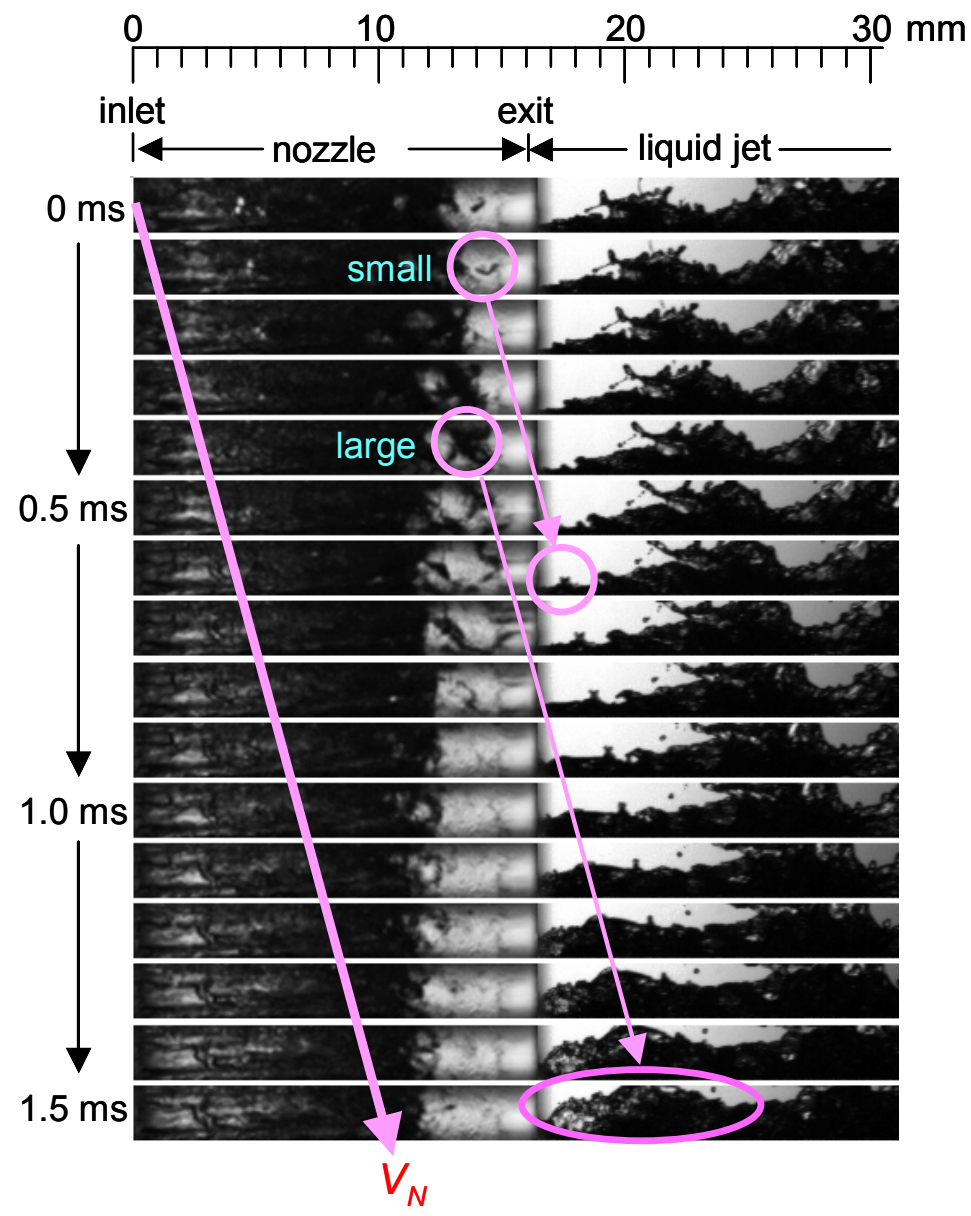

Fig. 15 A large cloud and a small cloud $\left(\sigma_{c}{ }^{\prime}=0.83,10000 \mathrm{fps}\right)$

\section{Conclusions}

Cavitation in a cylindrical nozzle and liquid jets discharged from the nozzle are simultaneously visualized using a high-speed camera to investigate the ligament formation 
induced by cavitation in the supercavitation regime. Three mirrors are used to capture the front view of liquid jet interfaces and the side view of cavitation clouds in a frame of the camera. The high-speed visualization confirms that the collapse of cavitation clouds near the nozzle exit induces the formation of ligaments not only for $2 \mathrm{D}$ nozzles but also for cylindrical nozzles. The visualization also finds that the collapse of a large cavitation cloud tends to induce the formation of a large ligament, while that of a small cloud causes a small ligament.

\section{Acknowledgements}

The authors would like to express their thanks to Mr. Kenji Isozaki of Kobe University. This study was supported by Grant-in-Aid for Scientific Research (No. 18560170) from the Japan Society for the Promotion Science (JSPS).

\section{References}

(1) Bergwerk, W., Flow Pattern in Diesel Nozzle Spray Holes, Proceedings of the Institution of Mechanical Engineers, Vol. 173, No. 25 (1959), pp. 655-660.

(2) Hiroyasu, H., Arai, M. and Shimizu, M., Break-up Length of a Liquid Jet and Internal Flow in a Nozzle, Proceedings of International Conference on Liquid Atomization and Spray Systems 91 (ICLASS 91) (1991), pp. 275-282.

(3) Chaves, H., Knapp, M., Kubitzek, A., Obermeier, F. and Schneider, T., Experimental Study of Cavitation in the Nozzle Hole of Diesel Injectors Using Transparent Nozzles, SAE Paper (1995), Paper No. 950290, pp. 645-657.

(4) Soteriou, C., Andrews, R., and Smith, R., Direct Injection Diesel Sprays and The Effect Cavitation and Hydraulic Flip on Atomization, SAE Paper (1995), Paper No. 950080.

(5) Collicott, S. H. and Li, H., True-scale True-Pressure Internal Flow Visualization for Diesel Injectors, SAE Paper (2006), Paper No. 2006-01-0890.

(6) Tamaki, N., Shimizu, M. and Hiroyasu, H., Enhancement of the atomization of a liquid jet by cavitation in a nozzle hole, Atomization and Sprays, Vol. 11, No. 2, (2001) pp. 125-137.

(7) Wu, P.-K., Miranda, R. F. and Faeth, G. M., Effects of Initial Flow Conditions on Primary Breakup of Nonturbulent and Turbulent Round Liquid Jets, Atomization and Sprays, Vol. 5, Iss. 2, (1995), pp. 175-196.

(8) He, L. and Ruiz, F., Effect of Cavitation on Flow and Turbulence in Plain Orifices for Highspeed Atomization, Atomization and Sprays, Vol. 5, No. 6, (1995) pp.569-584.

(9) Oda, T. and Yasuda, Y., Experimental Investigation on Structure of Cavitating Flow and Velocity Field inside a 2-D Hole Nozzle", CD-ROM of 9th International Conference on Liquid Atomization and Spray Systems (ICLASS 2003), (2003) ICLASS03-1205.

(10) Sou, A., Tomiyama, A., Hosokawa, S., Nigorikawa, S. and Maeda, T., Cavitation in a Two-Dimensional Nozzle and Liquid Jet Atomization (LDV Measurement of Liquid Velocity in a Nozzle), JSME International Journal, Series B, Vol. 49, No. 4, (2006) pp. 1253-1259.

(11) Sou, A., Hosokawa, S. and Tomiyama, A., Effects of Cavitation in a Nozzle on Liquid Jet Atomization, International Journal of Heat and Mass Transfer, Vol. 50, Iss. 17-18, (2007), pp. 3575-3582.

(12) Sou, A., Maulana, M. I., Isozaki, K., Hosokawa, S. and Tomiyama, A., Effects of Nozzle Geometry on Cavitation in Nozzles of Pressure Atomizers, Journal of Fluid Science and Technology, Vol. 3, No. 5, (2008), (in press).

(13) JSME, e.d., JSME Data Book, Hydraulic Losses in Pipe and Ducts, (2004) Maruzen.

(14) Tamaki, N., Flow in a Nozzle and Mechanism on Atomization of a Liquid Jet (in Japanese), Dr. Thesis, Hiroshima University, (1997). 